\title{
DEVELOPMENT OF AUTOPILOT FOR MICRO AERIAL VEHICLE USING STM32 MICROCONTROLLER
}

\author{
Manjunath B Kempar ${ }^{1}$, Rudresh M.D ${ }^{2}$ \\ ${ }^{I}$ M.Tech (VLSI Design and Embedded System), Department of Electronics and Communication Engineering, \\ Kalpataru Institute Technology Tiptur-572202, Karnataka, India \\ ${ }^{2}$ Associate Professor, Department of Electronics and Communication Engineering, Kalpataru Institute Technology \\ Tiptur-572202, Karnataka, India
}

\begin{abstract}
The performance of an autopilot controller has been an issue for industries over these days as they make use of less effective microcontroller. This limitation can be overcome by using FreeRTOS based implementation logic and by replacing less effective microcontroller with STM32 microcontroller. The prime objective of this paper is the development of FreeRTOS based autopilot controller using STM32.this autopilot controller comprises of Global Positioning System (GPS), Sensor Suite, external flash for data logging, Servo motor to control aileron, rudder and elevator action and MAVLink based transceiver in order to communicate between Micro Aerial Vehicle (MAV) and Ground Control station (GCS).this system focuses not only on device monitoring but also controlling it. The GCS comprises of Mission Planer software which is used to monitor the data received from MAV and can also control the flight mode from it. The Radio Frequency $(R F)$ joystick is used for MAV's flight control.
\end{abstract}

Key Words: Autopilot controller, Free Real time operating system (FreeRTOS), STM32, GPS, MAVLink, MAV, GCS, Mission Planer, RF.etc.......

\section{INTRODUCTION}

Since the concept of MAV was proposed a few decades ago, Micro Aerial Vehicles (MAVs) have attracted a lot of interest from researchers all over the world. Many kinds of MAV prototypes have been developed by universities and research laboratories. The primarily goal is to make the MAV have as much functions as possible and suitable for different missions.MAV can significantly reduce the risk to human life and minimize the economic damage in the scientific, military and civil application. The vertical takeoff and landing (VTOL) MAVs are particularly useful in missions such as surveillance and reconnaissance, homeland security, precision target location, signals intelligence, digital mapping, forest fire monitoring, and indoor rescuing. Since the miniaturization of Sensors, advances in communication devices and battery Technology, MAVs are becoming much smaller in size, Lighter In weight and can perform completely new missions such as operation at street level in urban environments [1]. Autopilots are systems to guide the UAVs in flight without Assistance from human operators. The autopilot technology of MAVs have become a hot research point in the field since the Constraints of their sizes and costs [2]. Nowadays, Technological advances in wireless networks and micro Electromechanical systems (MEMS) make it possible to use Small autopilots on MAVs [3]. These autopilots have characteristics of small size, low power, and high integration; basically meet the requirements of guiding a single $\mathrm{MAV}$ in all stages including take-off, ascent, descent, trajectory following, and landing.
This paper focuses on the design and development of autopilot suitable for Micro Aerial Vehicles (MAVs). The autopilot design solves the problems of autonomous control, navigation without the human intervention. Autopilot design for MAV's is designed to be very small and they usually have battery as their power source. The autopilot board has onboard processors and sensors. These sensors are usually MEMS (Micro Electro-Mechanical Systems), because such size and weight is always a limiting factor in MAV's. These sensors collect the flight data like acceleration, velocity, altitude, pressure and temperature. These parameters are fed to the onboard computer. The onboard computer also has a data link to a ground station called Ground Control Station (GCS) from where the user sends commands to the MAV. Based on these commands and the data collected the MAV flies and complete its mission. Other innumerous applications of MAV's have also been implemented. It also finds use in space missions. MAV's usually carry a camera, a night vision device, a GPS and if possible an autopilot. Autopilot is needed when the data link between the MAV and the ground station is broken, otherwise MAV would fall from the sky.

\section{SYSTEM DESIGN}

\subsection{Autopilot Controller}

The arm core present in the STM32F4 microcontroller provides the platform for complete Autopilot controller. Fig1 depicts the working of Autopilot controller. The Autopilot controller is used to control the trajectory of the MAV without requiring constant control by a human 
operator. Autopilot cannot replace human operator but help them in controlling the MAV and allowing them to focus on other operations such as monitoring the weather, trajectory and so on. The gains for autopilot were based on the flight parameters. Unfortunately mathematical model describing the flight dynamics of the MAV does not exit, so a trial and error approach has been adopted. The block diagram of autopilot controller is shown in Fig.1.

The system is designed such that if there is any deflection in the wings then the sensors detects and sends the signal to the autopilot controller. Then the auto pilot computer detects the signal and determines that the wings are not level. The autopilot controller generates PWM signal based on the sensor output and sends it to the servo motors that controls the aircrafts ailerons, elevators and rudder. This PWM signals will guide the servos in which direction and with which angle it has to rotate. The wings will move back to the level position if the ailerons, elevators and rudder are adjusted based the input PWM data. The autopilot controller removes the command to the servos when the position sensors detects that the wings are once again level.

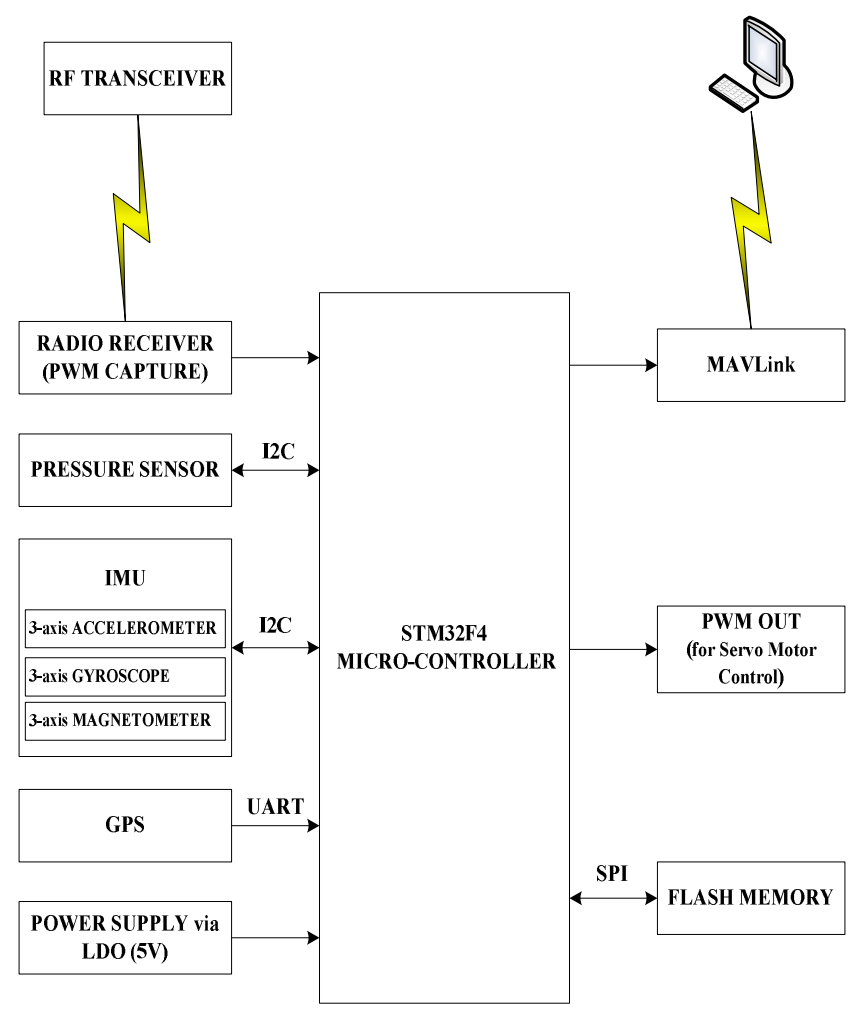

Fig.1 Block Diagram of Autopilot Controller.

\subsection{Hardware Design}

\subsubsection{STM32F4}

The STM32 F4-series is the first group of STM32 microcontrollers based on the ARM Cortex-M4F core. It has Digital Signal Processing (DSP) and floating point Unit (FPU). ARM Cortex-M4F core runs at a maximum clock rate of 84 / 168 / $180 \mathrm{MHz}$ Most oftnly controller work well with $168 \mathrm{MHZ}$. It supports standard embedded memories (SRAM is up to $196 \mathrm{~KB}$ out of which $128 \mathrm{~KB}$ on bus matrix and $64 \mathrm{~KB}$ on data bus dedicated for CPU usage. and $4 \mathrm{~KB}$ for battery backup for RTC) Up to $1 \mathrm{MB}$ Flash.

It also features standard and advanced high performance communication interfaces,

- $\quad$ An USB OTG-HS (High Speed) and USB OTG FS (Full Speed) with full speed compatibility.

- $\quad$ Three I2C (Inter Integrated Circuits).

- $\quad$ Three full duplex SPI (Serial Peripheral Interface)

- $\quad$ Two UARTs and four USARTs.

\subsubsection{Digital Pressure Sensor (BMP180)}

This sensor is used to measure the pressure which will be used to measure the altitude. The temperature measurement can also be performed using this sensor. The sensor is designed to be connected directly to a microcontroller of a mobile device via the $\mathrm{I} 2 \mathrm{C}$ bus. The pressure and temperature data has to be compensated by the calibration data of the E2PROM of the sensor. The pressure sensor consists of a piezo-resistive sensor, an analog to digital converter and a control unit with E2PROM and a serial I2C interface. It delivers the uncompensated value of pressure and temperature. The E2PROM has stored 176 bit of individual calibration data.

The 176 bit E2PROM is partitioned in 11 words of 16 bit each. These contain 11 calibration coefficients. Every sensor module has individual coefficients. Before the first calculation of temperature and pressure, the master reads out the E2PROM data. The BMP sensor will gives the Uncompensated Pressure (UP) and Uncompensated Temperature (UT). Pressure data is a 16 to 19 bit and Temperature data is a 16 bit.

The temperature data word UT, the pressure data word UP and the calibration data read out from the sensor are used to calculate the true pressure in steps of $1 \mathrm{~Pa}(=0.01 \mathrm{hPa}=$ $0.01 \mathrm{mbar}$ ) and temperature in steps of $0.1^{\circ} \mathrm{C}$. Figure 4.5 shows the application circuit of the temperature and pressure sensor.

With the measured Pressure $\mathrm{P}$ and the pressure at sea level Po, the altitude in meters can be calculated with the international barometric formula:

$$
\text { altitude }=44330 *\left(1-\left(\frac{P}{P 0}\right)^{\frac{1}{5.255}}\right) \ldots \ldots . . m
$$

\subsubsection{Magnetometer Sensor (HMC5840)}

Magnetometers are devices that measures magnetic fields and it can sense where the strongest magnetic force is coming from. This sensor is a surface-mounted, multi-chip module designed for low-field magnetic sensing with a digital interfaces for application such low-cost compassing and magnetometer. The sensor include our state-of-the-art, high-resolution series magneto-resistive sensors plus an 
ASIC containing amplification, automatic degaussing strap drivers, offset cancellation, and a 12-bit ADC that enables $1^{\circ}$ to $2^{\circ}$ compass heading accuracy. The I2C serial bus allows for easy interface. This is a $3.0 \times 3.0 \times 0.9 \mathrm{~mm}$ surface mount 16-pin leadless chip carrier (LCC). Applications for this sensor include Mobile Phones, Notebooks, Consumer Electronics, Auto Navigation Systems, and Personal Navigation Devices. These anisotropic, directional sensors feature precision in-axis sensitivity and linearity. Magnetometer full scale range is \pm 8 gauss.

\subsubsection{Motion Processing Unit (MPU9150) Sensor}

The 9DoF-IMU sensor is a multi-chip module (MCM) consisting of two dies integrated into a single LGA package. One die houses the 3-Axis gyroscope and the 3Axis accelerometer. Communication with all registers of the device is performed using $\mathrm{I} 2 \mathrm{C}$ at $400 \mathrm{KHz}$. Additional features include an embedded temperature sensor and an on-chip oscillator with $\pm 1 \%$ variation over the operating temperature range.

\subsubsection{MEMS Gyroscope with 16-bit ADCs and}

\section{Signal Conditioning}

The IMU includes a 3-Axis vibratory MEMS rate gyroscope, which detect rotations about the X-, Y-, and ZAxes. When the gyro is are rotated about any of the sense axes, the Coriolis Effect causes a vibration that is detected by a capacitive pickoff. The resulting signal is amplified, demodulated, and filtered to produce a voltage that is proportional to the angular rate. This voltage is digitized using individual on-chip 16-bit Analog-to-Digital Converters (ADCs) to sample each axis. The full-scale range of the gyro sensor may be digitally programmed to $\pm 250, \pm 500, \pm 1000$, or \pm 2000 degrees per second (dps). The ADC sample rate is programmable from 8,000 samples per second, down to 3.9 samples per second, and user selectable low pass filters enable a wide range of cut-off frequencies.

\subsubsection{Three-Axis MEMS Accelerometer with 16-}

\section{bit ADCs and Signal Conditioning}

The IMU's 3-axis accelerometer uses separate proof masses for each axis. Acceleration along a particular axis induces displacement on the corresponding proof mass, and capacitive sensors detect the displacement differentially. The IMU's architecture reduces the accelerometer's susceptibility to fabrication variations as well as to thermal drift. When the device is placed on a flat surface, it will measure $0 \mathrm{~g}$ on the $\mathrm{X}$ - and $\mathrm{Y}$-axes and $+1 \mathrm{~g}$ on the $\mathrm{Z}$-axis. The accelerometer's scale factor is calibrated at the factory and is nominally independent of supply voltage. Each sensor has a dedicated sigma-delta ADC for providing digital outputs. The full scale range of the digital output can be adjusted to $\pm 2 g, \pm 4 g, \pm 8 g$, or $\pm 16 g$.

\subsubsection{Global Positioning System (GPS)}

The GPS is a satellite based navigation system that provides location and timing information in all weather conditions, anywhere on or near the Earth surface where there is an unobstructed line of sight to four or more GPS satellites. The 3DR GPS MTK V2.0 is a compact Patch on Top (POT) GPS Module. This POT type GPS receiver provides high tracking capabilities with high in position and speed accuracy performances, and highly sensitive. This module supports up to 66 channels, and designed for small form factor devices.

\subsection{Software Design}

\subsubsection{STM Cube MX}

STM32CubeMX is a free graphical configuration tool with low level code generator for STM32 ARM Cortex-M microcontrollers. STM32CubeMX will facilitate Microcontroller Unit selection, suggest pin assignments and check for consistency, create start up code and generate configuration files for middleware. It also generates IDE ready projects that includeSTM32Cube drivers and middleware.

\subsubsection{FreeRTOS}

FreeRTOS is areal time kernel for embedded devices. Real time kernel is a microkernel and is distributed under the GNU General Public Licence (GNU GPL) with an optional exception. It is having Avery small memory footprint, low overhead and a very fast execution. Preemptive or cooperative scheduler operation can either be configured.This also includes task scheduling and kernel calls for both semaphore and queue operations.

\section{METHODOLOGY}

The system that is autopilot controller which is placed in the aircraft should be able to acquire the data from the sensors and store them in the flash and should be able to send the data to the ground control station (GCS) via radio frequency controlled 3DR transceiver module which is present at both aircraft and at GCS .The flight movement can be controlled by using radio frequency controlled joystick. The pwm signal generated by the autopilot controller is used to control the ailerons, elevator and rudder.

This paper focuses mainly on design and implementation of autopilot controller. Design of autopilot board is done using Cadence Orcad tool which includes MCU with 3 sensors (i.e., Pressure sensor (BMP 180), IMU (MPU 9150) and magnetometer (HMC 5840)) interfaced using I2C protocol. The SPI protocol is used to interface the external flash for data logging.

The data from the 3 sensors are recorded and used to calculate pitch, roll and yaw in degrees, pressure in $\mathrm{hPa}$, temperature in ${ }^{\circ} \mathrm{C}$, altitude in meters, and magnetic field in gauss which in turn stored in the flash. Now the calculated 
pitch, roll and yaw angles are compared with the threshold value then If there is any change in the angle detected or the change in the position of the wing then autopilot controller detect the variation and generate the pwm signal accordingly based on the variation and it will be given to the servo motor placed in order to control the principal axis of the flight i.e., to control the ailerons, rudder, and elevators. This is depicted in Fig.2.

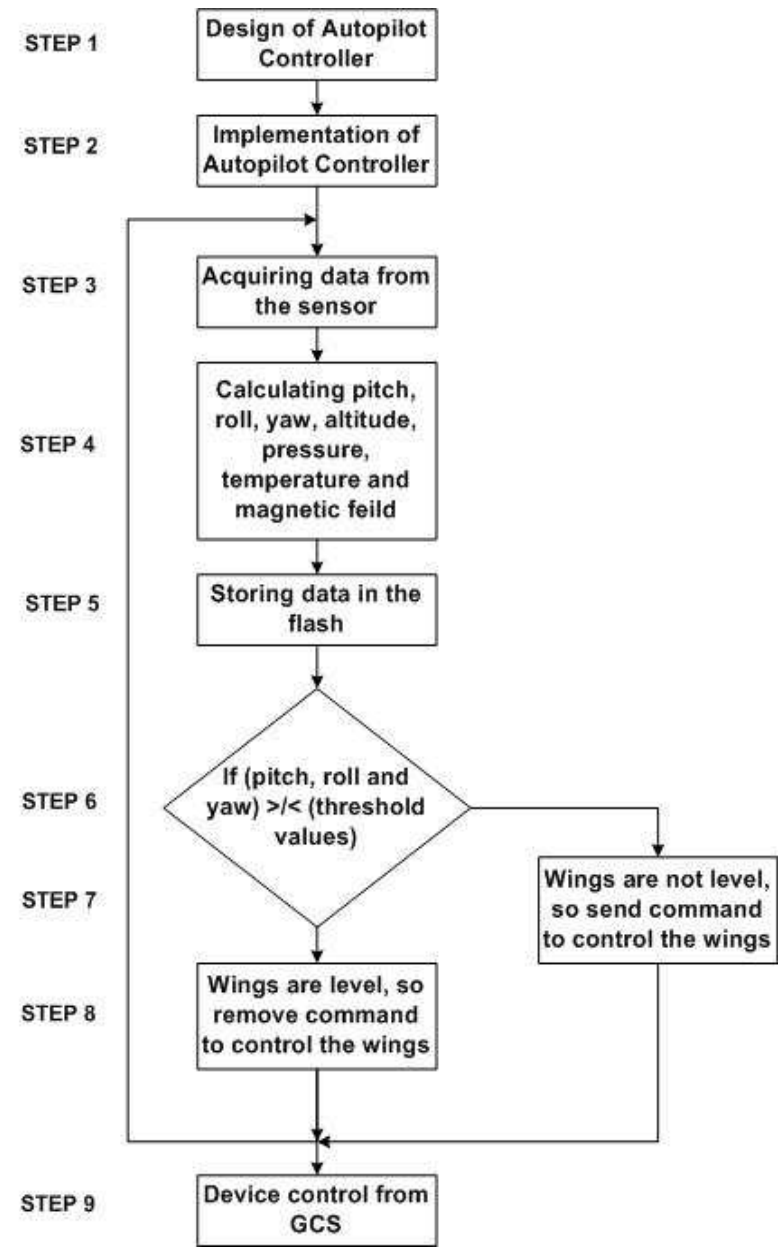

Fig.2 Flow chart of the working of the Autopilot Controller

\section{EXPERIMENTAL RESULTS}

The results obtained by the integration of the sensors, i.e. the pressure sensor for measuring pressure, temperature and altitude. The magnetometer sensor for measuring magnetic field and the 9DoF-MPU sensor for measuring out the accelerometer value in $\mathrm{x}, \mathrm{y}, \mathrm{z}$ direction and gyro meter value to calculate pitch, roll and yaw .GPS is used to measure the position of MAV and location.Are discussed. These sensors are interfaced to the STM32F4 evaluation board using I2C and UART protocol and the results are viewed and analyzed using logic analyzer. The setup of the experiment is shown in Fig 3.
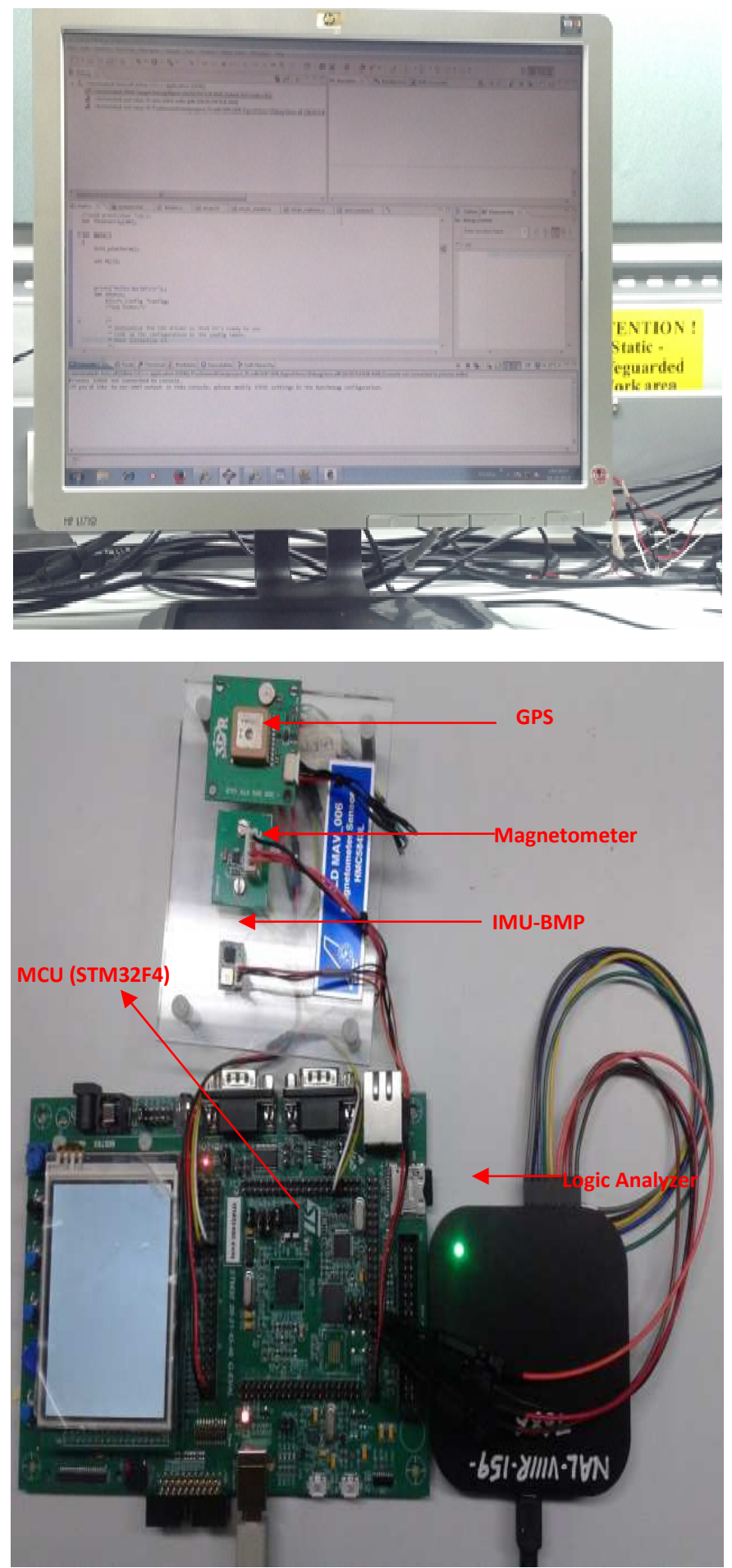

Fig.3 Work bench setup using STM32

Results obtained by the Integration of the pressure sensor with STM32 Evaluation Board :

In experimental setup, by using I2C protocol we interfaced all three sensors. These are pressure sensor, the magnetometer sensor and the 9DoF-IMU sensors. The I2C lines are initialized with the required mode, duty cycle, clock speed etc. To ensure that the communication is happening with the required slave device the chip id or the device id of the slave is checked. The result obtained by reading the register I2C_address_reg0 is shown in Fig 4. 


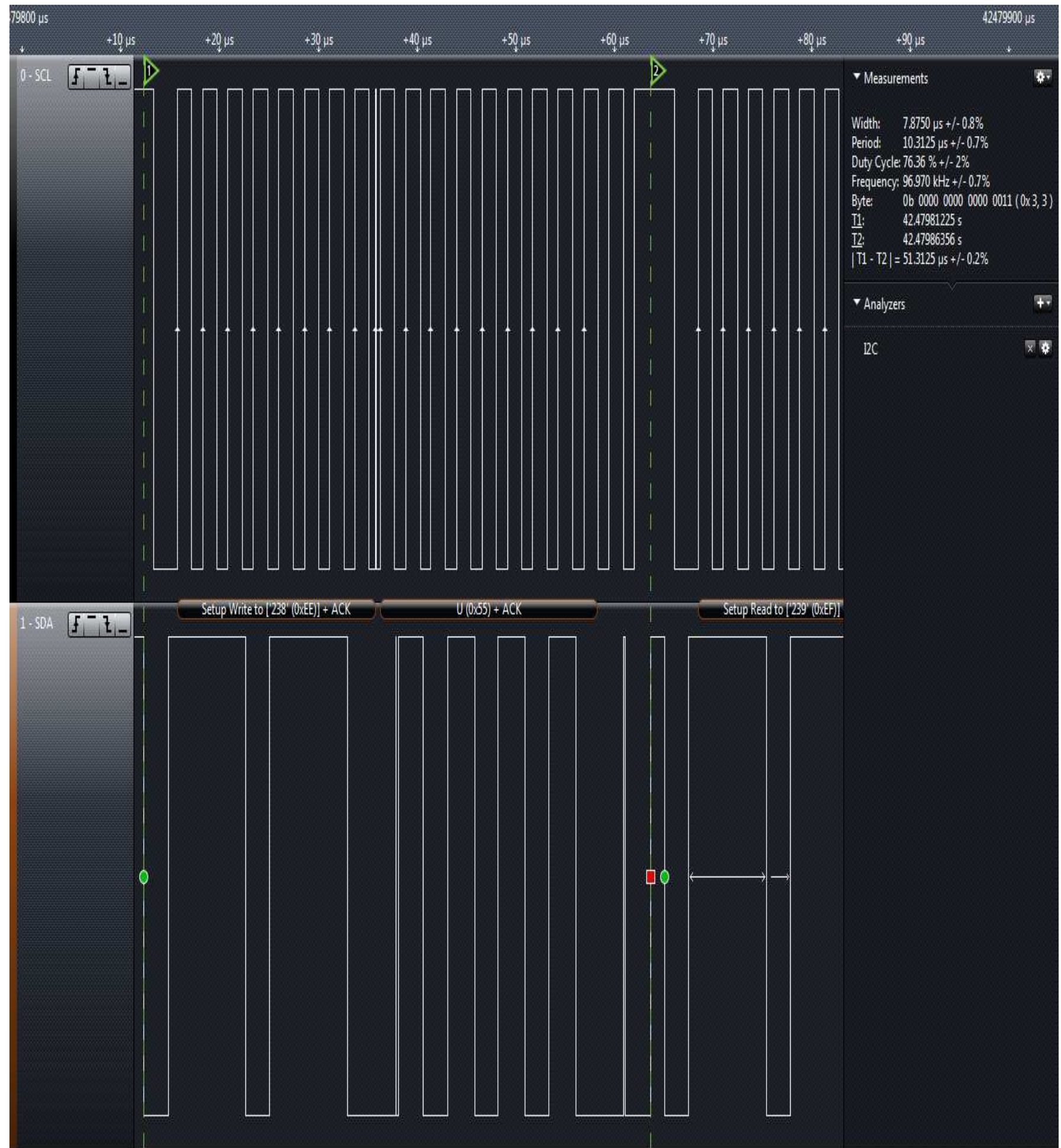

Fig4: Reading data from BMP shown in logical analyzer

Results obtained by the Integration of the magnetometer sensor STM32 Evaluation Board:

The hard ware set up for Integration of the magnetometer sensor with master device and the logical analyzer is shown in Figure2. The I2C lines are initialized with the required mode, duty cycle, clock speed etc. To ensure that the communication is happening with the required slave device the chip id or the device id of the slave is checked. The magnetometer sensor used has three registers to check the identity of the device i.e. identification register A with the value of $0 \times 48$, identification register $B$ with the value of $0 \times 34$ and identification register $\mathrm{C}$ with the value of $0 \times 33$. The identification register $\mathrm{A}(0 \mathrm{x} 0 \mathrm{~A})$, identification register $\mathrm{B}(0 \mathrm{x} 0 \mathrm{~B})$ and the identification register $\mathrm{C}(0 \mathrm{x} 0 \mathrm{C})$ are read out and the data obtained is shown in Fig 5. 


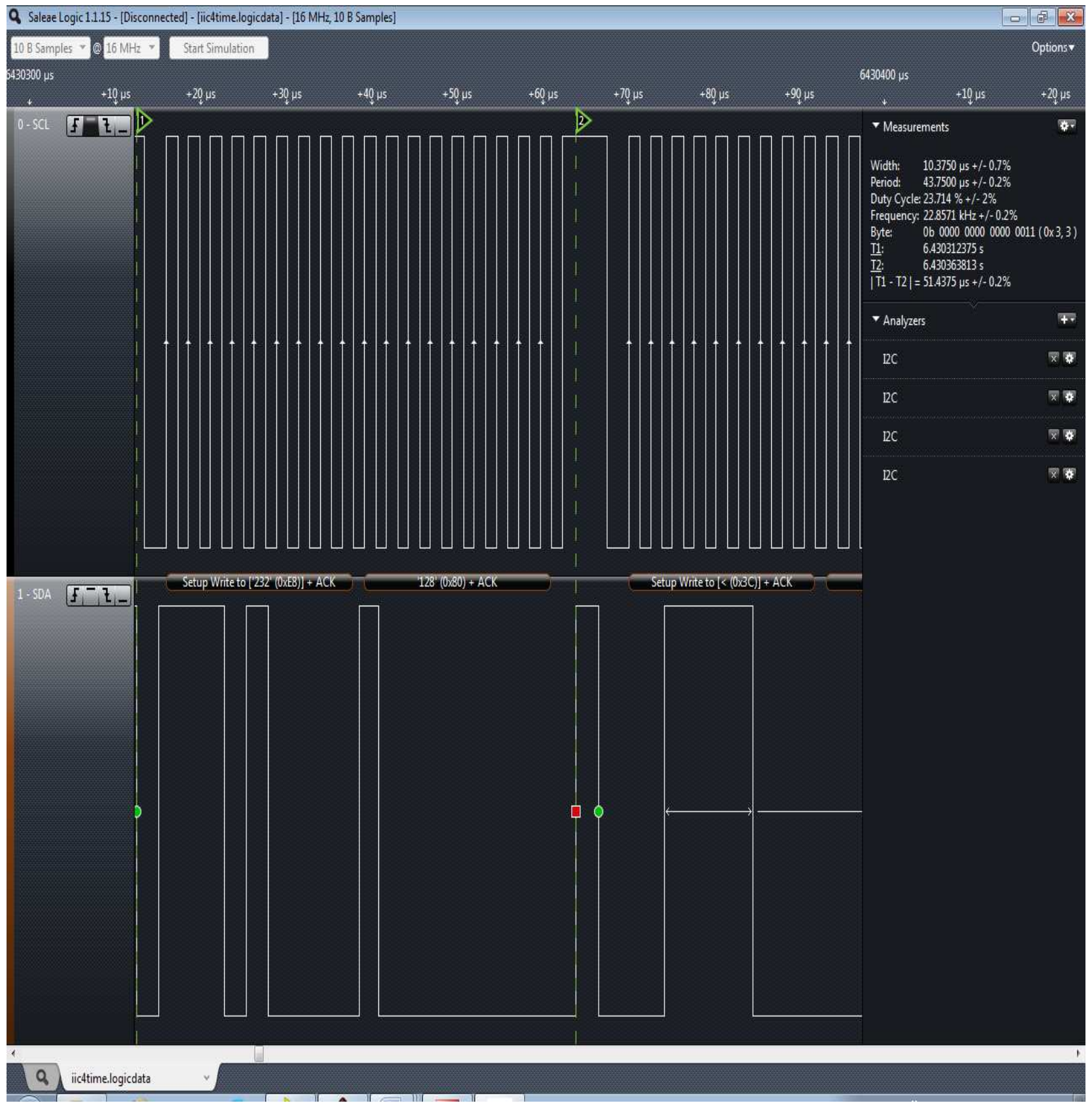

Fig5: Reading data from Magnetometer shown in logical analyzer

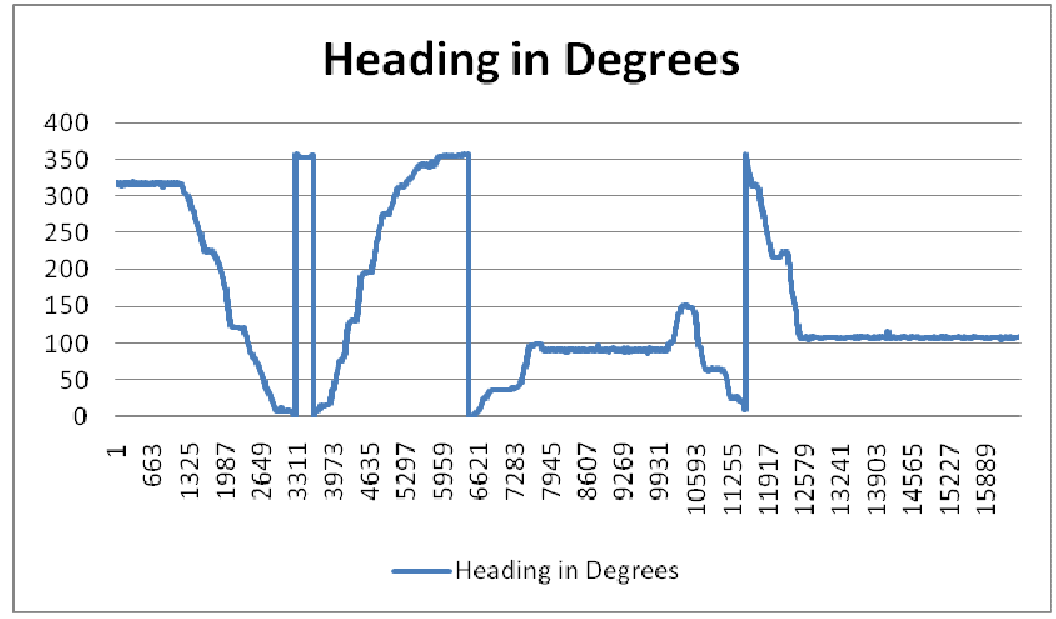

Fig 6: results obtain by the magnetometer 
Results obtained by the Integration of the IMU sensor STM32 Evaluation Board:

The I2C lines are initialized with the required mode, duty cycle, clock speed etc. To ensure that the communication is happening with the required slave device the chip id or the device id of the slave is checked. The 9DOF-IMU sensor used has a register called WHO_AM_I to verify the identity of the device. The WHO_AM_I register with address of 0x75 has a value 0x68 stored in it. I2C Communication between Magnetometer and STM32 board captured in logical analyzer is shown in Fig 7.

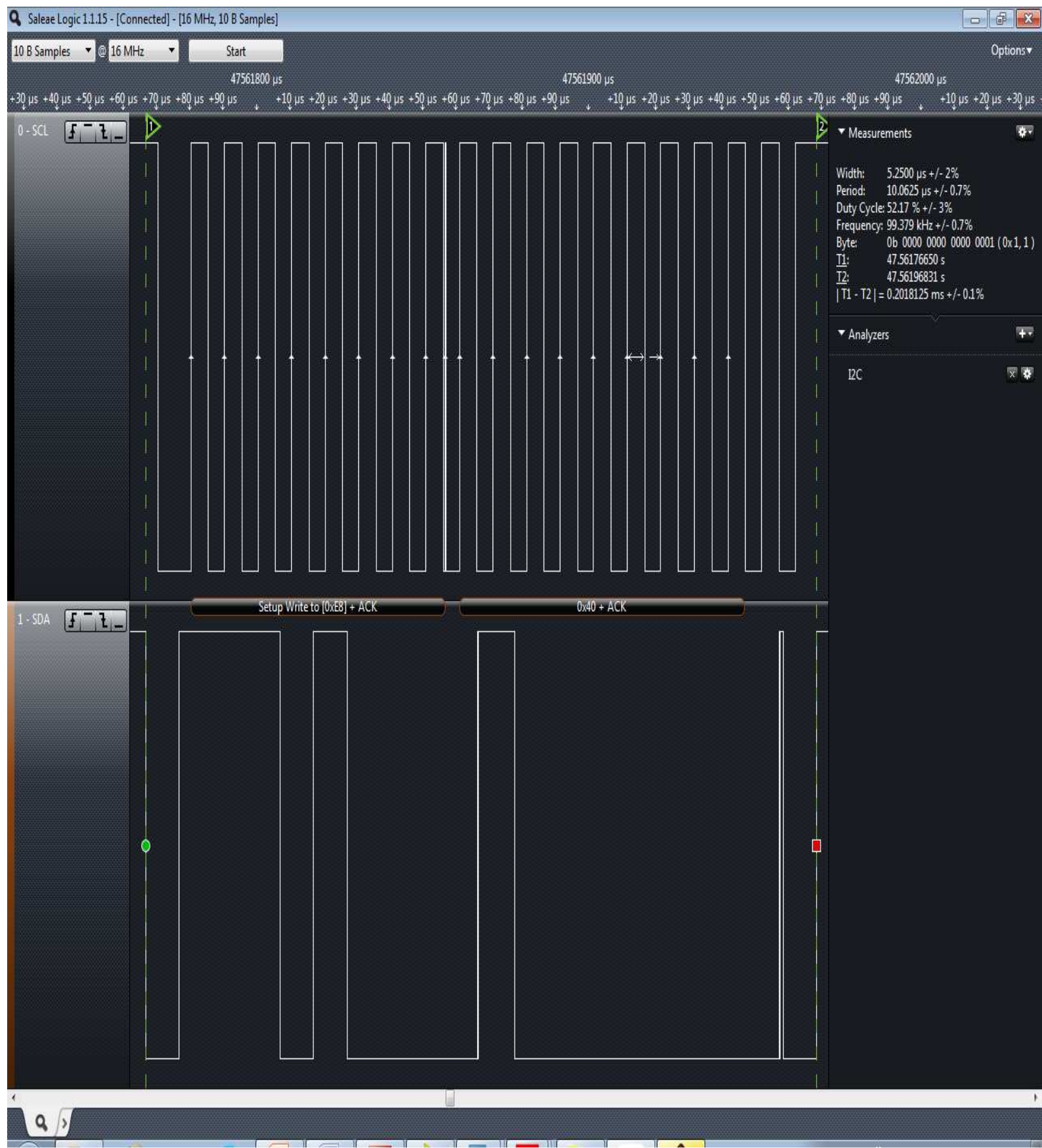

Fig 7: Reading data from MPU shown in logical analyzer 


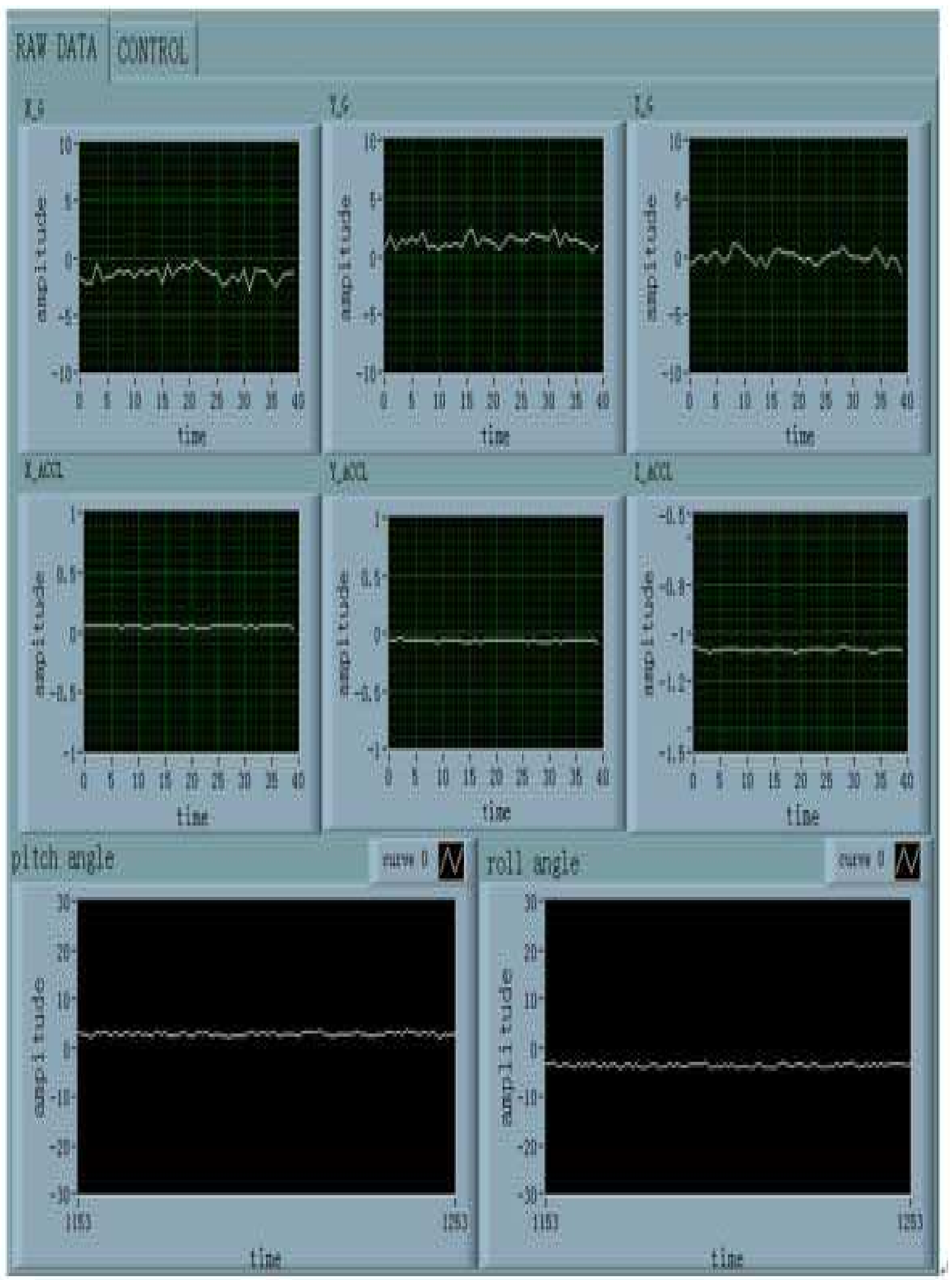

Fig 8: results obtain by the MPU sensor (Pitch, roll, yaw) 
The retrieved data from the BMP is shown in Fig 7.

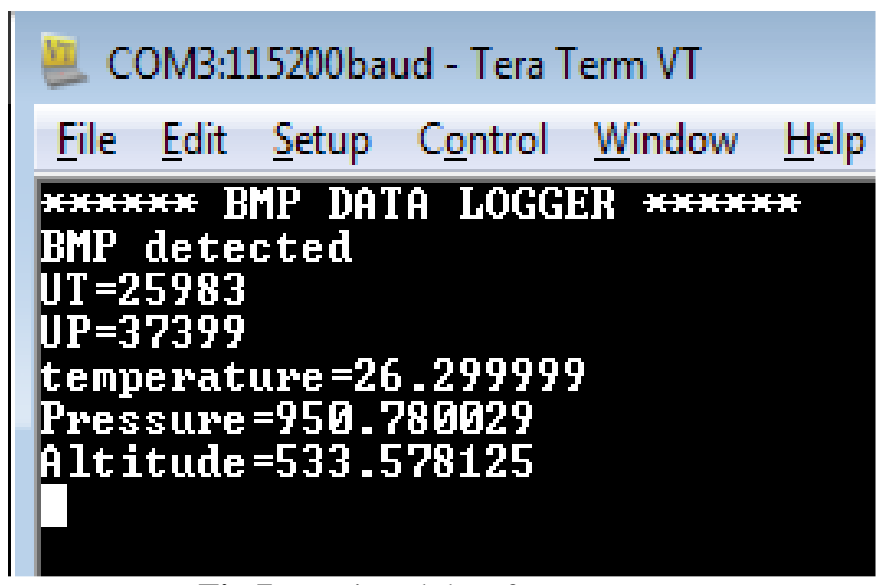

Fig 7: Retrieved data from BMP

Depending upon these sensor values stored in external flash memory the autopilot controller will generates the PWM (Pulse Width Modulation) signals to control the MAV with required condition.

\section{SYSTEM MERITS}

\subsection{Existing Work}

The method of using conventional autopilot controller has a limitation in processing capability and also lags in producing reactive output. Conventional autopilot controller demands large amount of memories and area which lead also to an increase in system cost. The comparison between existing and the proposed system is shown in Table.1.

\subsection{Proposed Work}

The problem of Size, cost and performance can be overcome by using the FreeRTOS based STM32 autopilot controller, as it does well in all the domains in which the conventional systems fail.

\section{CONCLUSION}

The rapid development in the field of avionics demands for an efficient implementation of unmanned Micro aerial vehicle. The Real time implementation of STM32F407IGHXbased auto pilot controller is an effective solution in terms of performance, size and cost when compared to that of the traditional method of using Micro aerial vehicle.

\section{ACKNOWLEDGEMENTS}

I would like to thank the Aerospace Electronics and System Division (ALD), CSIR-NAL, Bangalore, for providing the computational resources and services used in this paper. I would also like to acknowledge my internal guide $\mathrm{Mr}$. RUDRESH M.D, Associate Professor, Department of ECE, Kalpataru Institute of Technology, Tiptur for providing us an opportunity to carry out my project in a prestigious organization.

\section{REFERENCES}

[1] Fu,Li,Luo,Han ."Autopilot design for control of Rotorcraft Micro Aerial Vehicles (RMAVs) Swarm." 978-1-4799-3322, IEEE, 2014

[2] Stingu,Emanuel, and Frank L. Lewis."A hardware platform for research in helicopter uav control."In Unmanned Aircraft Systems, pp. 387-406. Springer Netherlands, 2009.

[3] Pestana, J., Mellado-Bataller, I., Sanchez-Lopez, J. L., Fu, C.,Mondragón, I. F., \& Campoy, P. A General Purpose Configurable Controller for Indoors and Outdoors GPS-Denied Navigation for Multirotor Unmanned Aerial Vehicles. Journal of Intelligent \& Robotic Systems, 2014, 73(1-4), 387400.

[4] Chao, Haiyang, Yongcan Cao, and Yang Quan Chen. "Autopilots for small fixed-wing unmanned air vehicles: A survey." In Mechatronics and Automation, 2007. ICMA 2007. International Conference on, pp.

[5] "Design of a Miniature, Multi-Directional Optical Flow Sensor for Micro Aerial Vehicles" Daniel Watman and Hideaki Murayama, International Conference on Robotics and Automation Shanghai International Conference, 2011, Shanghai, China.

[6] J. Metge, R. Megret, A. Giremus, Y. Berthoumieu, C. Mazel 'Dynamic magnetic field compensation for micro UAV attitude estimation' International Conference on Unmanned Aircraft Systems, 2013

[7] Xiangtong Kong, Chunping Wang, Shuying Sun, FeiGao, 'A Method of Digital to Shaft-angle Converting Using ARM series MCU\&CPLD' Fifth International Symposium on Computational Intelligence and Design,2012.

[8] Hines L.L., Arabagi V., Sitti M., 'Free flight simulations and pitch and roll control experiments of a sub-gram flapping-flight micro aerial vehicle',IEEE International Conference Robotics and Automation, 2011.

[9] S. Winkler, M. Buschmann, L. Kruger, H.-W. Schulz, P. Vorsmann, 'Multiple Sensor Fusion for Autonomous Mini and Micro Aerial Vehicle Navigation',IEEE-2011.

[10] Jinjun Rao, TongyueGao, Zhen Jiang and Zhenbang Gong, 'Flight Control System of a Robotic Portable Unmanned Aerial Vehicle' IEEE-2010.

\section{BIOGRAPHIES}

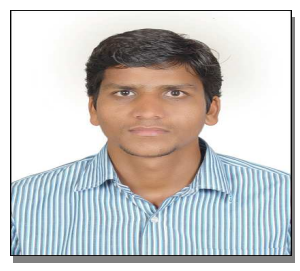

Manjunath B Kempar was born on $20^{\text {th }}$ MAY,1991.He received the B.E degree in Electronics and Communication Engineering from KVG College of Engineering Sullia D K, VTU-Belgaum,Karnataka in 2013, currently pursuing Master of Technology in VLSI Design and Embedded System from Kalpataru Institute of TechnologyTiptur,VTUBelgaum,Karnataka.His areas of interest are Embedded System Design, Real Time Operating System,VLSI Design, 
microcontroller Architecture such as ARM-Cortex M0,M3,M4 processors, MSP430. etc

Email:manju.kempar@gmail.com

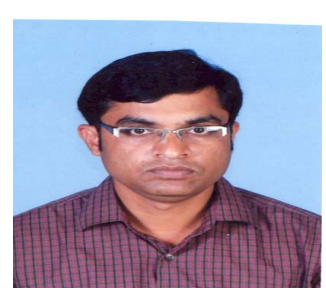

Rudresh M D, was born on $1^{\text {st }}$

June 1974, He received the B.E degree in Electronics and Communication Engineering from Bangalore University, Bangalore Karnataka in 2000.received M.Tech degree in Power Electronics from BMS College of Engineering, Bangalore in 2006.from 2000 to 2002 he was a Design Engineer at Future Techno design, Bangalore. From 2002 to 2004 he was lecturer at GTTC Kudal Sangama, Bagalkot District. From 2006 onwards and Currently he is an Associate Professor in Department of Electronics and Communication Engineering, Kalpataru Institute of Technology Tiptur. Currently pursuing $\mathrm{PhD}$ Programme at JNTU Hyderabad,Telangana. His research interests includes Biometrics,Biomedical and Speech Processing,Image Processing,DSP,Embedded system Design.

Email: rudresh_md@rediffmail.com 